\title{
Mortalidad Perinatal en el quinquenio 1985 a 1989 en la sección de ginecología y obstetricia del Hospital Militar C.
}

\author{
Dr.: Jairo de la Cruz S. *
}

\section{RESUMEN}

La tasa de mortalidad perinatal al considerarse como uno de los indicadores más sensibles del proceso reproductivo nos permite evaluar la calidad de atención dispensada al binomio madre-hijo. Se estableció dicha tasa en el quinquenio 1985 a 1989 en la sección de obstetricia y ginecología del Hospital Militar Central en el 19.69 por mil. La edad promedio de la madre fue de 29 años, el $59.85 \%$ de las muertes ocurrieron en etapa pretérmino; el $70.81 \%$ de los recién nacidos tuvieron peso superior a los 1.500 gramos.

La hipertensión arterial inducida por el embarazo y la amenaza de parto pretérmino son las complicaciones del embarazo que se asocian en mayor porcentaje a la mortalidad; el parto pretérmino fue la alteración del parto encontrada en mayor numero en los componentes ante e intraparto.

Las complicaciones del recién nacido se establecieron como nacidos pretérmino en el $58.9 \%$ y asociados a malformaciones congénitas en un $24.5 \%$.

De acuerdo con la clasificación internacional de 999 causas de mortalidad perinatal, las malformaciones congénitas ocuparon el primer lugar, seguidas de síndrome de dificultad respiratoria.

PALABRAS CLAVES: Embarazo, Mortalidad perinatal.

\section{SUMMARY}

The perinatal mortality rate being considered as one of the most sensible index in the reproductive process so it allows us to evaluate the quality of the assistance given to the binomial mother-so. It was established such rate in the quinquennium 1985 - 1989 at the Obstetrics and Gynecology Department of the Militar Hospital in 19.69/1000. Mother's age average was of the 29 years; the $59.85 \%$ of deaths occurred during the prematured stage; $70.81 \%$ of the new borns had weight superior to the 1.500 grams.

The arterial hypertension induced by the pregnancy and the prematured labor threatening are the pregnancy complications that are associated in a high percentage to the mortality; the prematured labor was the alteration given a great number in the pre and post-parturition components.

The complications for the new born were established as prematured borns in the $58.9 \%$ and associated with congenital malformations in an $24.5 \%$.

According to the international classification of 999 reasons of perinatal mortality, the congenital malformations occupied the first place followed by the breathing difficulty.

KEY WORDS : Pregnancy, Perinatal mortality. 


\section{INTRODUCCION}

Es conocido el significado que posee el estudio de las tasas de mortalidad perinatal en cuanto a la calificación de la calidad de atención obstétrica, y como, si este resultado es analizado en forma periódica nos brinda la oportunidad de detectar y corregir los errores cometidos en dicha atención (18).

La base inicial de este trabajo fue la información recolectada para el estudio cooperativo institucional en la ciudad de Bogotá sobre mortalidad perinatal y el estudio de mortalidad perinatal en 21 instituciones de Colombia, auspiciado por COLCIENCIAS y la Federación Colombiana de Sociedades de Obstetricia y Ginecología.

\section{ANTECEDENTES Y JUSTIFICACION}

Según se ha logrado establecer, la mortalidad perinatal es uno de los indicadores más sensibles de la calidad del proceso reproductivo $(18,35)$, allí radica la importancia de establecer periódicamente este parámetro.

Los estudios a nivel local, en instituciones de Bogotá, informan tasas promedio de 18.09 por mil, con fluctuaciones entre el 34.25 y el 4.5 por mil, para el año 85 (35).

A nivel nacional, en 21 instituciones del país, para el año 87 la tasa encontrada fue del 23.8 por mil, fluctuando entre 44.69 y 1.75 por mil, dependiendo del nivel de atención. En este informe nuestra entidad ocupa un puesto intermedio con tasa de 24.23 por mil en dicho año (36).

La tasa encontrada en nuestra nación en los años 85 a 87 se dieron en países desarrollados de Europa en el año 1966 (24), así en Inglaterra, Polonia y Alemania Oriental, la tasa fluctuaba entre 25 y 26 por mil; Austria tenía 29.8 por mil y la Secretaría de Salud de Bogotá informó una tasa de 29.70 en los años 83-84. La tasa del estudio interinstitucional de Bogotá en 1985 del 18.09 la presentó Suecia (18.9) en 1966. Según la OMS la mortalidad perinatal en países del tercer mundo es de 4 a 7 veces mayor que en países desarrollados (3).

La información estadística disponible en el servicio de ginecología y obstetricia del Hospital Militar se remontaba al año de 1974, cuando se realizó un análisis retrospectivo de octubre de 1963 , fecha de inicio de labores del departamento, a marzo de 1973. La tasa de mortalidad perinatal hallada en esa revisión se estableció en 22.4 x 1000, sobre 15792 partos atendidos (27).
Cada día y con mayor fundamento algunos autores cuestionan la utilidad de las sofisticadas unidades de cuidado intensivo obstétrico y neonatales en países de bajos recursos; dando al cuidado prenatal mayor importancia para disminuir la mortalidad perinatal (10).

El bajo peso al nacer se ha considerado como la causa más directa del mayor índice de mortalidad perinatal $(1,10,13,16,31,35,36,37)$ y al trabajo de parto pretérmino el que más contribuye a nacimientos de menos de 2500 gr. $(5,13,16,30,35,36,37)$. La ruptura prematura de membranas, la infección urinaria, la bacteriuria asintomática y el embarazo múltiple son los problemas obstétricos que con mayor frecuencia se asocian al parto pretérmino $(6,13,14,18,21,31)$.

Otros factores, en menor frecuencia, son fuentes de mortalidad perinatal; unos, de origen fetal, como anomalías congénitas, macrosomía, retardo del crecimiento intrauterino, sensibilización Rh. Otros, de origen materno, como abruptio de placenta, placenta previa, preeclampsia, diabetes, multiparidad, embarazos en adolescentes y en mujeres mayores de 35 años ( 1 , $5,10,15,28$ ).

Si conocemos las causas que condicionan la muerte perinatal deberíamos desarrollar estrategias tendientes a reducir la magnitud del problema. Por ello, se hace necesario el uso de instrumentos que permitan obtener la mayor información posible y la adopción de modelos de clasificación de la causa básica de la defunción. De igual manera, si hacemos uso rutinario de este tipo de información, nos permitirá canalizar los esfuerzos en áreas específicas, en donde podremos disminuir la mortalidad, evitando así la dispersión de acciones en forma innecesaria en áreas donde no se obtendrá beneficio.

Con base en estas consideraciones, el propósito es conocer en forma consistente la tasa de mortalidad perinatal en el servicio de obstetricia y ginecología del Hospital Militar Central durante el quinquenio 1985 a 1989, de igual manera, identificar las causas más importantes que contribuyen a ella.

\section{MATERIAL Y METODOS}

\section{Tipo de estudio}

Retrospectivo con análisis descriptivo.

\section{Población}

Se tomó la totalidad de muertes perinatales ocurridas desde el 1o. de enero de 1985 a diciembre 31 de 1989, en los servicios de obstetricia y ginecología y de pediatría del Hospital Militar Central. 


\section{Recolección de la información}

Se realizó en el formulario de registro de defunción perinatal diseñado para aportar la información al estudio interinstitucional auspiciado por la FECOLSOG. Además, se contabilizaron todos los nacimientos durante el período con el fin de calcular la tasa de mortalidad perinatal.

\section{Glosario de términos}

Se utilizaron las definiciones y términos recomendados por el Centro Latinoamericano de Perinatología y Desarrollo Humano (CLAP) $(31,32)$.

\section{Mortalidad Perinatal}

Toda muerte del producto ocurrida a partir de la semana 28 de gestación y antes del séptimo día de nacido (168 horas). En caso de no conocimiento de la edad gestacional se utiliza como parámetro todo producto muerto de 1.000 gramos de peso o más.

\section{Tasa de Mortalidad Perinatal}

Número de mortinatos de 1.000 o más gramos, más número de muertes de 1.000 o más gramos antes del séptimo día de nacido (168 horas) sobre el número total de nacidos vivos y muertos de 1.000 o mạ́s gramos al nacer, por mil.

\section{RESULTADOS}

Se analizaron 139 formularios, que corresponden a la totalidad de muertes perinatales ocurridas en el período lo. de enero de 1985 a diciembre 31 de 1989.

\section{Tasa de mortalidad perinatal}

En el quinquenio analizado se atendieron 6986 partos para un total de 7064 recién nacidos, la mayor tasa se presentó en el año 1987, con 28.14 por mil, y la menor en 1988 con 15.37 por mil. La tasa de mortalidad perinatal global para el período en cuestión fue del 19.69 por mil (Gráfica 1). La tasa del decenio 63-73 (22.4 por mil) y la del presente quinquenio 85-89 (19.69 por mil) se ven en la Gráfica 2.

\section{Edad de la Madre}

El promedio de edad de la madre fue de 29 años. Un poco más de la mitad de los casos suceden en madres con edad de 23 a 32 años, 74 casos, para un $54.41 \%$; el porcentaje de embarazos en adolescentes es bajo, $2.94 \%$, y en mujeres mayores a 37 años llega al $10.29 \%$ (Gráfica 3).

\section{Estado civil de la madre}

De 124 casos, sólo 7, o sea el $5.65 \%$ no tenían una unión estable, o un compañero permanente (Gráfica 4).
GRAFICA 1

\section{TASA QUINQUENIO 1985-1989}

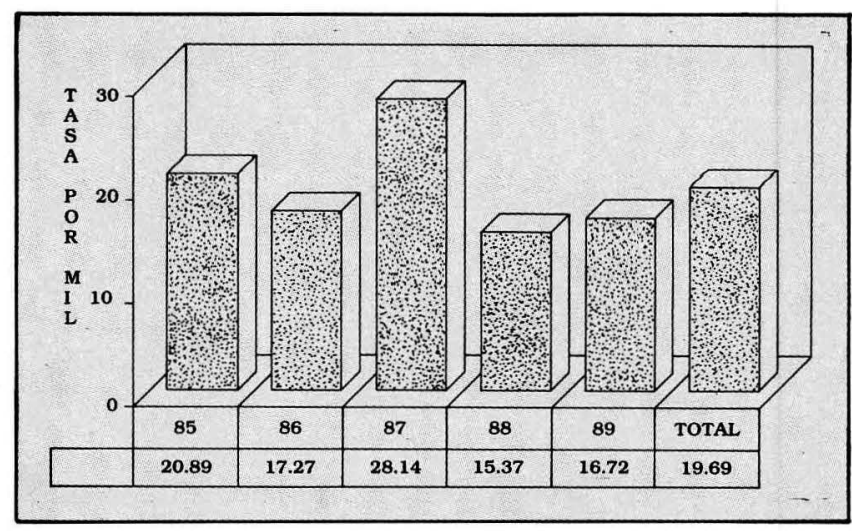

GRAFICA 2

TASA COMPARATIVA

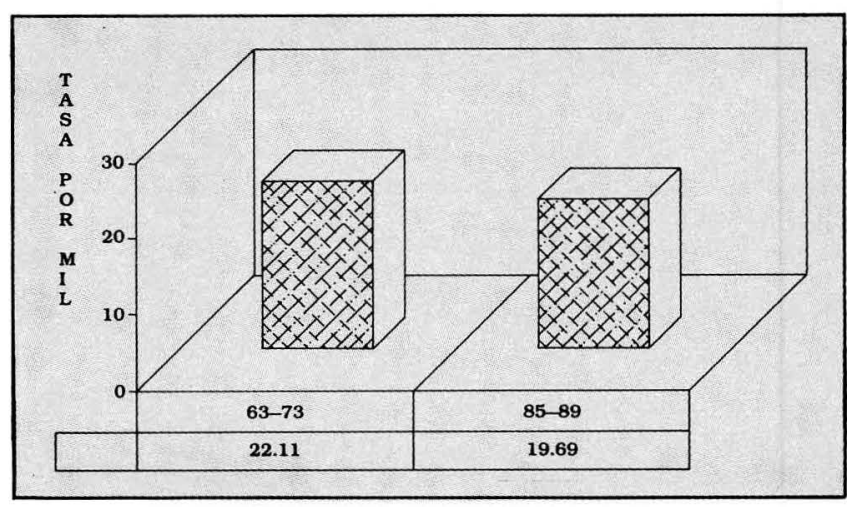

GRAFICA 3

\section{EDAD MATERNA QUINQUENIOS}

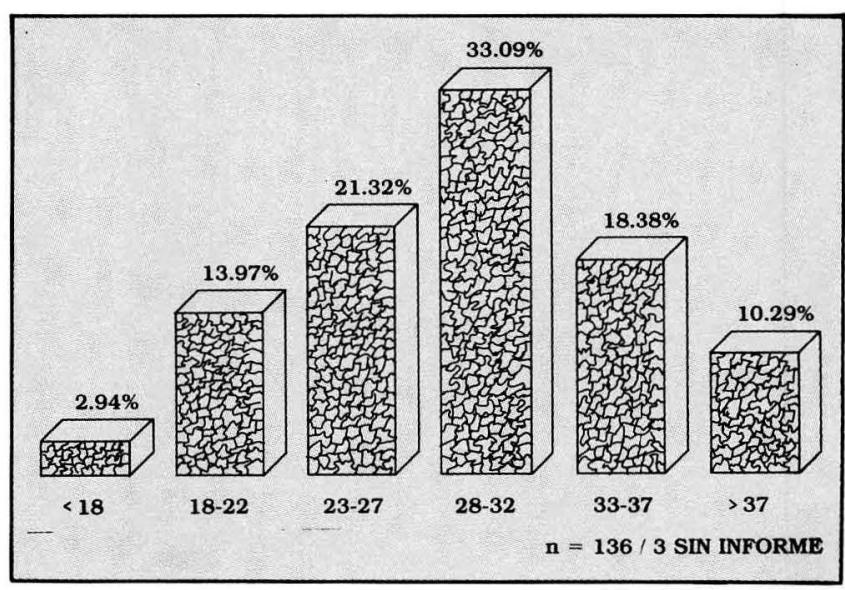


GRAFICA 4

ESTADO CIVIL DE LA MADRE

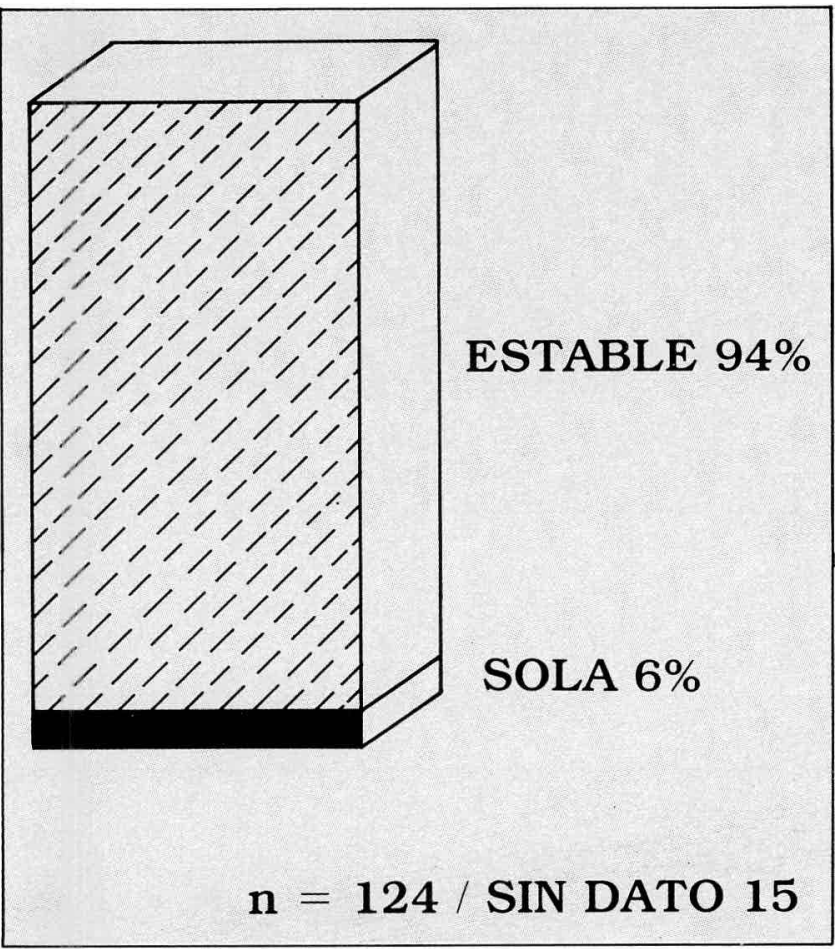

Distribución de la muerte perinatal según el número de embarazos previos

Dos de cada tres pacientes tenían de uno a cuatro partos, únicamente el $2.99 \%$ tenían cinco o más partos. El 31.34\% eran primigestantes (Gráfica 5).

GRAFICA 5

\section{EMBARAZOS PREVIOS}

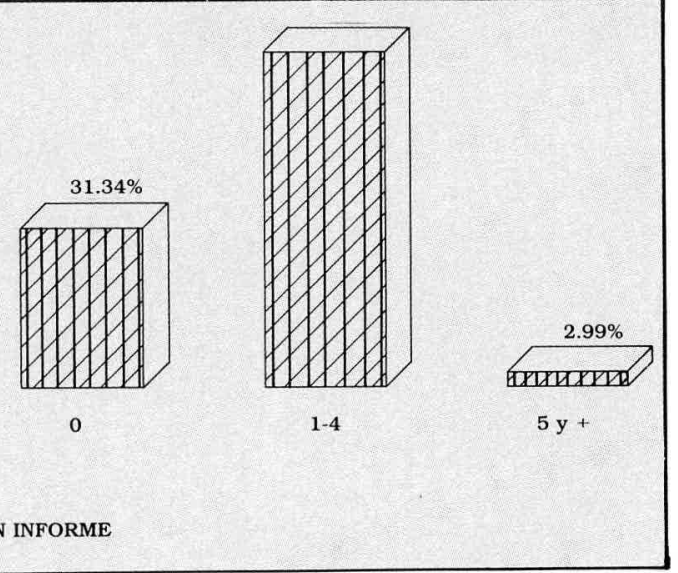

Distribución de la muerte perinatal según atención prenatal

$22.32 \%$ de las pacientes carecieron de atención en su embarazo, al resto, $77.68 \%$ se le realizó al menos un control (Gráfica 6)

GRAFICA 6

CONTROL PRENATAL

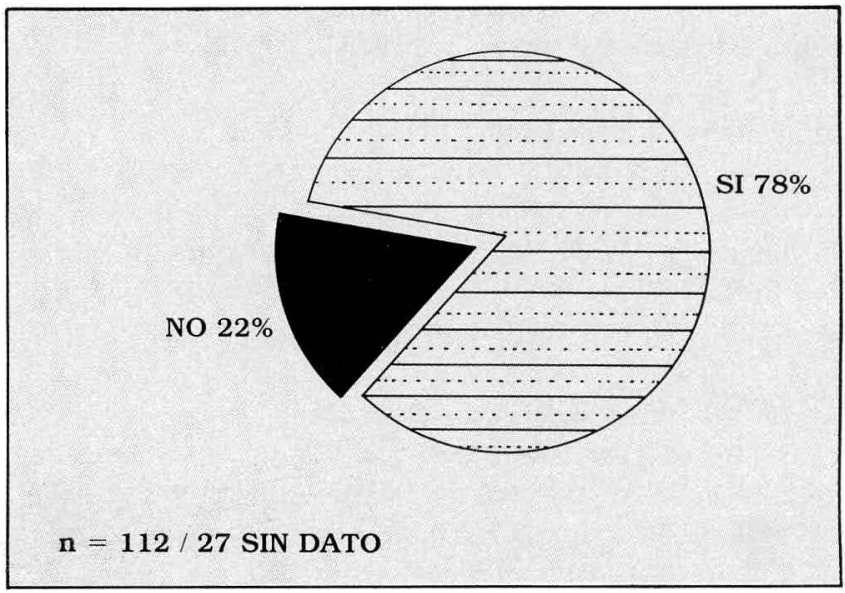

Muertes perinatales según la edad gestacional

Se encontró un $59.85 \%$ de muertes en etapa pretérmino, sólo una paciente, $0.73 \%$ cursó embarazo mayor a las 42 semanas. En el $39.42 \%$ de ellas la gestación se encontraba a término (Gráfica 7).

GRAFICA 7

EDAD GESTACIONAL

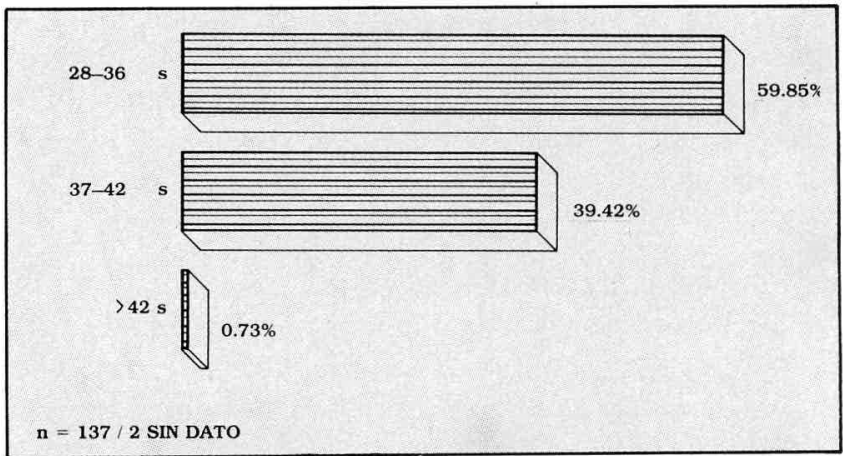

Distribución de las muertes perinatales según duración del trabajo de parto

Una de cada tres pacientes presentó trabajo de 2 a 6 horas $(34.53 \%)$; otra, no tuvo trabajo de parto $(33.81 \%)$; y la otra, o tuvo trabajo menor de 2 horas o mayor de 7 horas (Gráfica 8). 
GRAFICA 8

DURACION DEL TRABAJO DE PARTO

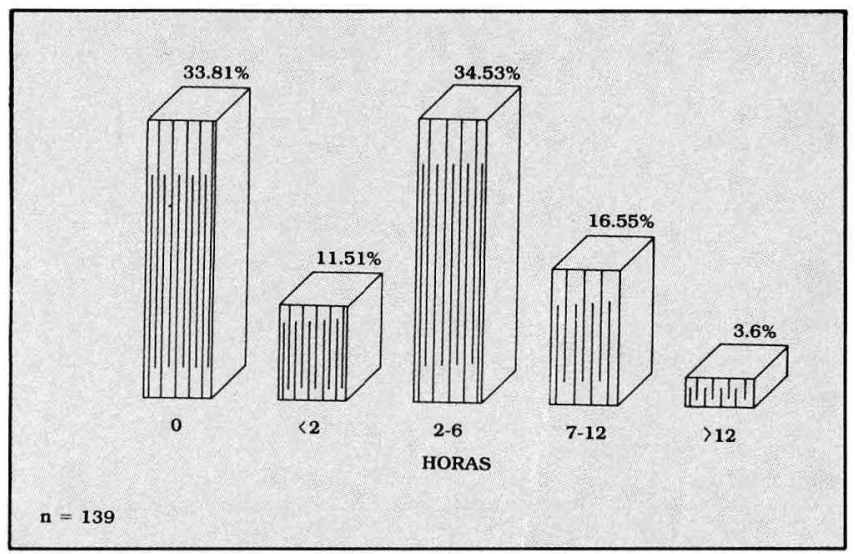

Forma de terminación del embarazo

69 pacientes se intervinieron quirúrgicamente, el $49.64 \%$, y 66 presentaron parto vaginal espontáneo, $47.48 \%$, cuatro partos se instrumentaron, $2.88 \%$ (Gráfica 9).

GRAFICA 9

\section{TERMINACION DEL EMBARAZO}

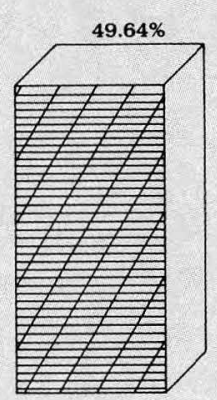

CESAREA

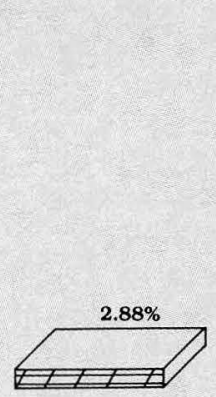

VAG. INTERV.

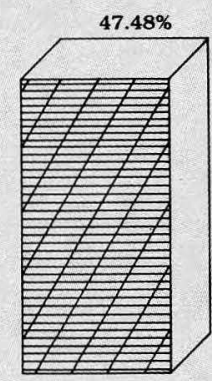

VAG. ESPON. $\mathrm{n}=139$

Muertes perinatales según presentación del recién nacido

La presentación cefálica predominó con un total de 116 casos el $83.45 \%, 16$ nacieron en presentación podálica, $11.5 \%$; y, siete tuvieron otro tipo de presentación, 5.04\% (Gráfica 10).

\section{Mortalidad perinatal según peso al nacer}

El quinquenio nos permite apreciar a recién nacidos con peso superior a los 1.500 gr en el $70.81 \%$ y menores de 1.000 gr 40 niños para un 29.2\% (Gráfica 11).
GRAFICA 10

SEGUN PRESENTACION DEL RN

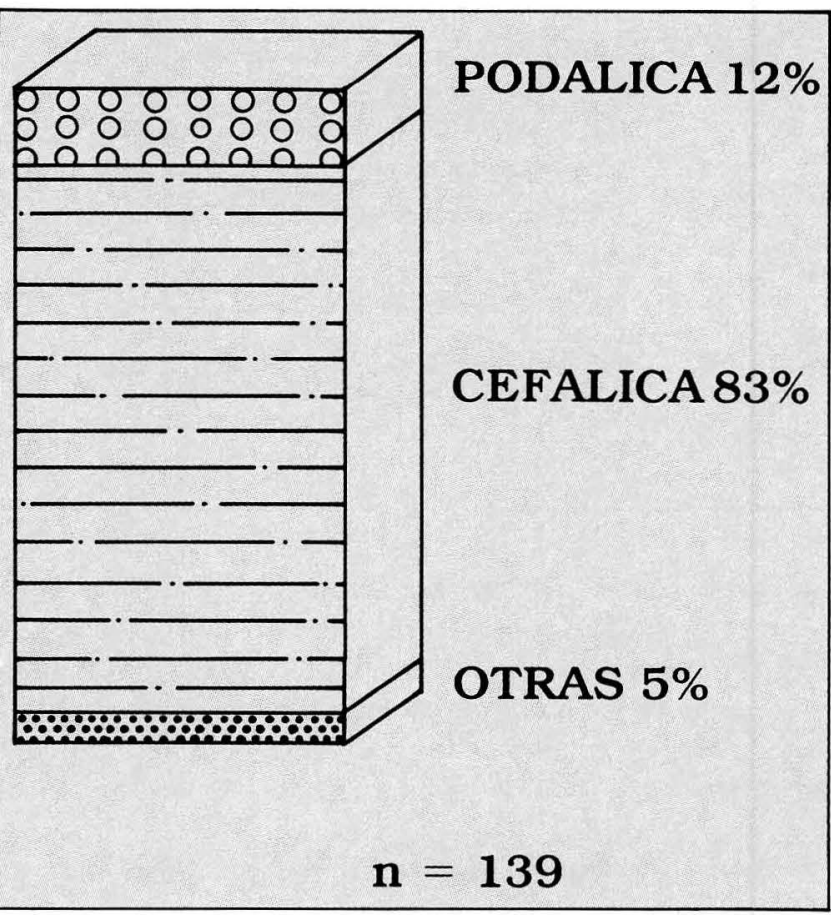

GRAFICA I 1

SEGUN PESO AL NACER

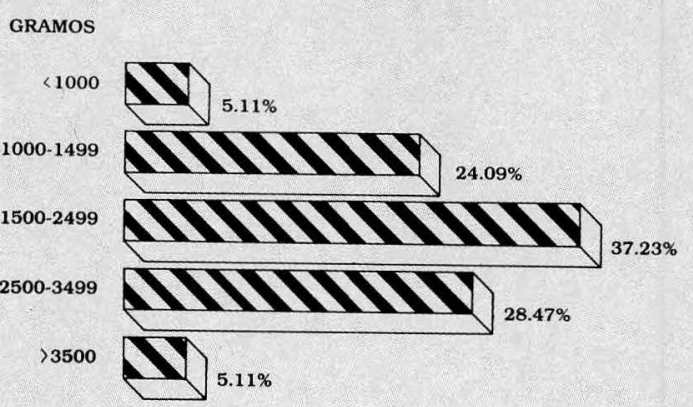

$\mathrm{n}=136 / 3$ SIN DATO

Promedio de peso al nacer según componentes de la mortalidad perinatal

El conjunto muestra una distribución uniforme en los tres componentes de la mortalidad perinatal y un promedio final de 2.067 gr (Gráfica 12).

\section{Muertes perinatales según sexo}

Se apreció un mayor número de fetos masculinos muertos, el $55.40 \%$, el $40.29 \%$ fueron de sexo femeni- 
GRAFICA 12

PROMEDIO DE PESO AL NACER SEGUN COMPONENTES DE LA MORTALIDAD

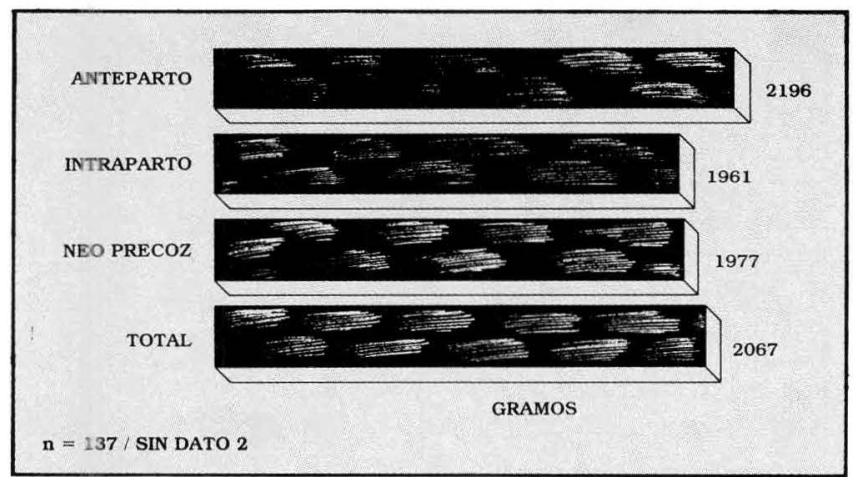

no, en 6 casos $(4.32 \%)$ no fue posible determinar el sexo. Los hallazgos están de acuerdo con lo descrito en la literatura. Al analizar el índice de masculinidad, vemos que por cada 100 mujeres que fallecen, mueren 137 hombres; según la fórmula: $\mathrm{IM}=$ No. Hombres / No. Mujeres x 100 (Gráfica 13).

GRAFICA 13

\section{SEXO DEL RECIEN NACIDO}

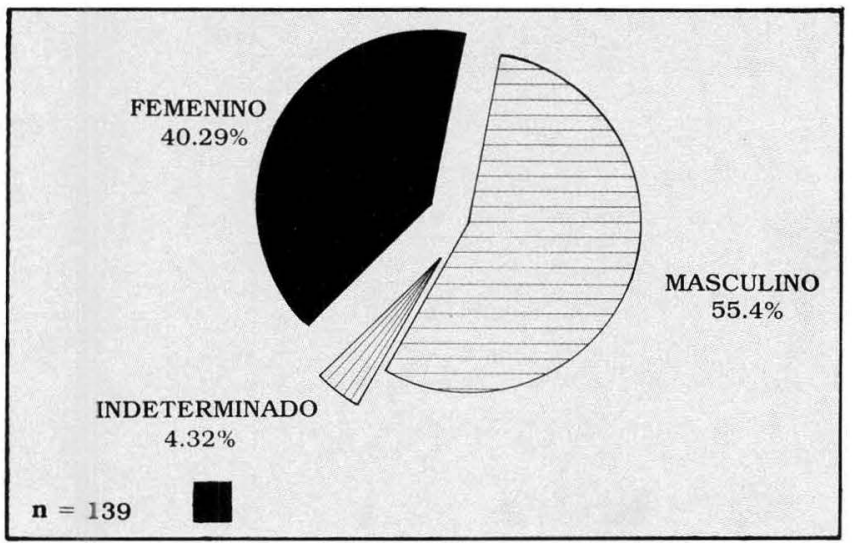

Mortalidad perinatal según apgar al minuto y cinco minutos

Menos de la mitad, el $41.73 \%$ de los casos tuvieron un apgar de 0 , es decir nacieron muertos, y el $17.27 \%$ el apgar fue de 7 o más. De total de nacidos vivos (81) once fallecieron antes de los cinco minutos, mientras que el $53.09 \%$ presentó en ese momento un apgar de 7 o más (Gráficas 14-15).

Distribución de las muertes perinatales según sus componentes

Al analizar el quinquenio la mortalidad neonatal precoz fue superior al 50\%. 79 casos, $56.83 \%$ sucedie-
GRAFICA 14

APGAR AL MINUTO

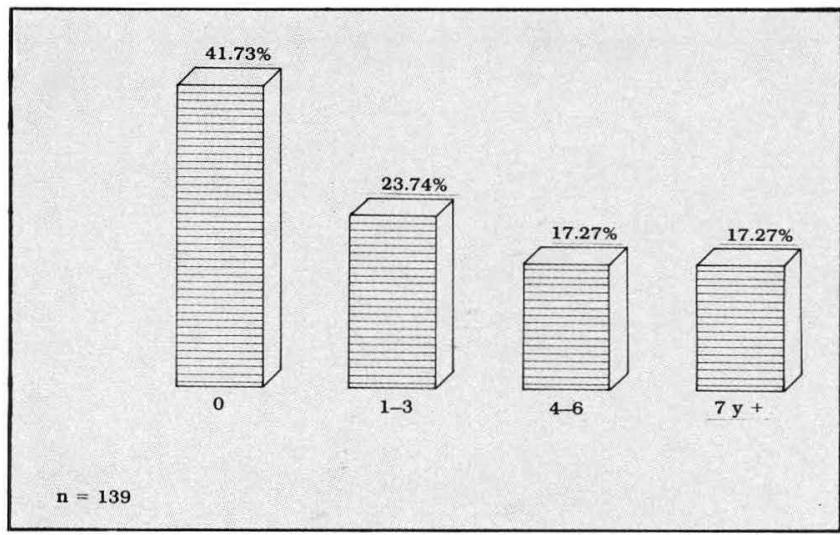

GRAFICA IS

APGAR AL 5 MINUTO

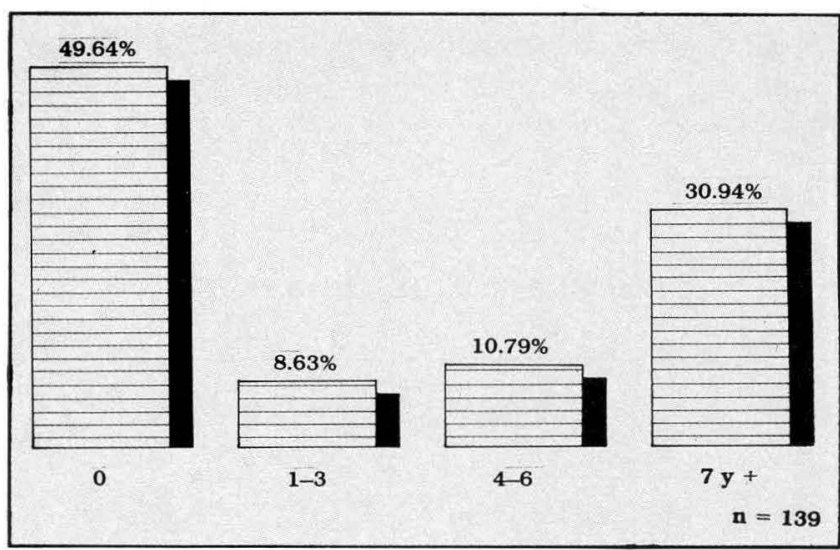

ron en esta fase; 53 casos, $38.13 \%$ en la etapa anteparto y 7 , el $5.04 \%$, en el intraparto. La mortalidad fetal tardía, de acuerdo con lo anterior, es de $43.16 \%$ (Gráficas 16-17).

GRAFICA 16

\section{SEGUN COMPONENTES}

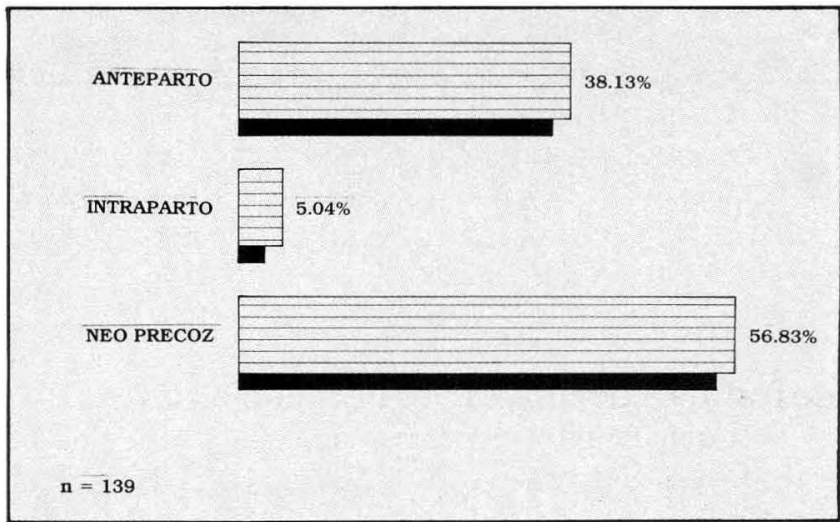


GRAFICA 17

SEGUN COMPONENTES

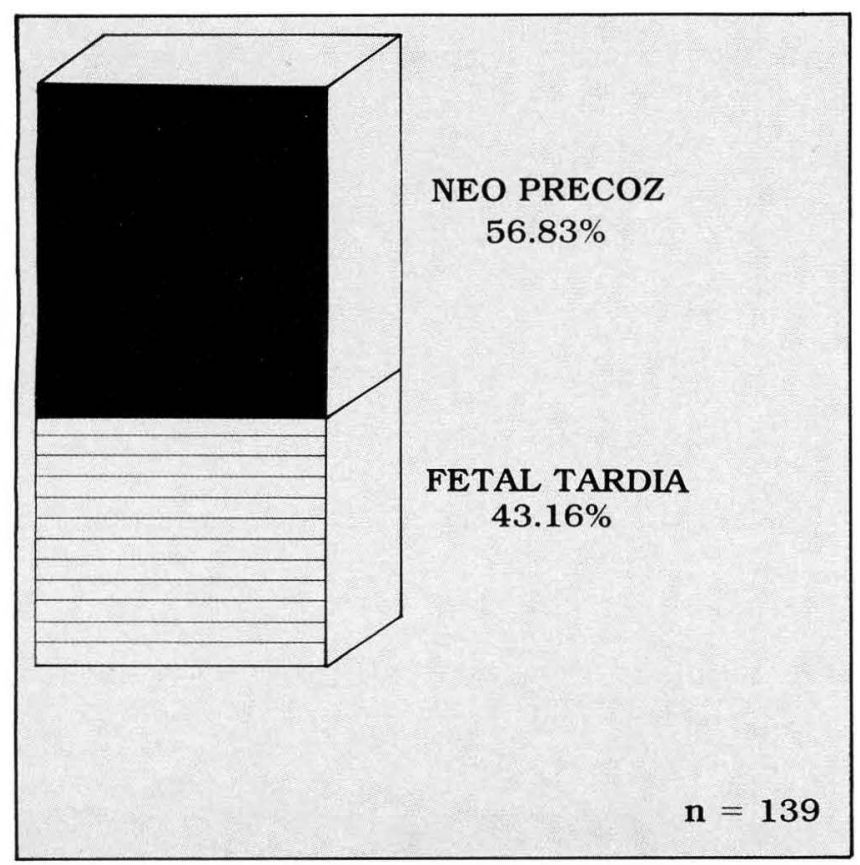

Autopsia al recién nacido

A pesar de la importancia que tiene el diagnóstico patológico, a sólo una de cada tres muertes perinatales se les realizó este estudio. El $62.59 \%$ de los casos no tuvo estudio postmorten (Gráfica 18).

GRAFICA 18

\section{AUTOPSIA AL RECIEN NACIDO}

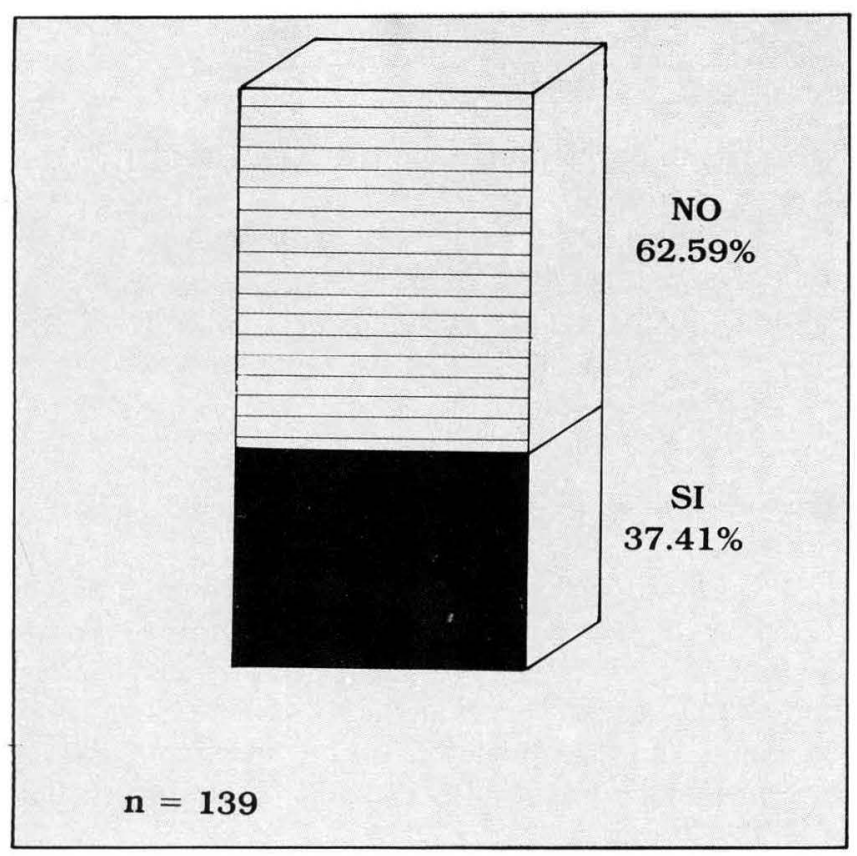

\section{Complicaciones del embarazo asociadas con la mortalidad perinatal}

En el transcurrir de los cinco años las primeras complicaciones maternas asociadas a la mortalidad neonatal fueron la amenaza de parto pretérmino, la hipertensión inducida por el embarazo y el abruptio. Las primeras diez causas se observan en la Tabla 1, la primera con 20 casos, $14.4 \%$, la ocupó la hipertensión inducida por el embarazo, seguida por la amenaza de parto pretérmino con 15 casos, $10.8 \%$. Otras causas, en orden, corresponden al abruptio, la ruptura prematura de membranas y el polihidramnios.

TABLA 1

COMPLICACIONES DEL EMBARAZO ASOCIADAS CON LA MORTALIDAD PERINATAL

\begin{tabular}{|rlcr|}
\hline No. & \multicolumn{1}{c}{ Complicación } & Total & * \% \\
\hline 1 & Hipertensión inducida EMB & 20 & 14.4 \\
2 & Amenaza parto pretérmino & 15 & 10.8 \\
3 & Abruptio de placenta & 14 & 10.0 \\
4 & Ruptura prematura de MEM & 9 & 6.5 \\
5 & Polihidramnios & 9 & 6.5 \\
6 & Embarazo Múltiple & 8 & 5.8 \\
7 & Incompatibilidad RH & 7 & 5.0 \\
8 & Retardo crecimiento I.U & 6 & 4.3 \\
9 & Gran Multípara > 5 partos & 4 & 2.9 \\
10 & Oligoamnios & 3 & 2.2 \\
\hline
\end{tabular}

$* \mathrm{n}=139$

La suma de los casos de amenaza de parto pretérmino y ruptura prematura de membranas da un porcentaje de 17.3 cifra que se convertiría en la primera causa asociada a la mortalidad situación íntimamente relacionada con partos pretérmino y síndrome de dificultad respiratoria del recién nacido (Tablas 2-3).

\section{Afecciones maternas concurrentes asociadas con la mortalidad perinatal según sus compo- nentes}

En el componente anteparto la tercera parte de las muertes se debieron a hemorragia de la segunda mitad del embarazo, un poco menos, el $11.3 \%$ a trastornos hipertensivos inducidos por la gestación. En el intraparto menos de la mitad, $42.8 \%$ presentaron igualmente sangrado después de la semana 20 de la gestación, la ruptura de membranas ocurrió en el $14.2 \%$ de las muertes e igual porcentaje se encontró en el polihidramnios. En el componente neonatal precoz los trastornos hipertensivos, la hemorragia y la ruptura de membranas, en su orden, se asociaron a la mortalidad (Gráfica 19).

\section{Complicaciones de parto asociadas con la mortalidad perinatal}

El parto pretérmino fue complicación de primer orden, parto por cesárea y de pelvis ocuparon los siguientes lugares en el lapso de los cinco años. Dos de 
tres complicaciones del parto corresponden a partos pretérmino y uno de cada dos se intervino mediante operación cesárea (Tabla 2).

TABLA 2

COMPLICACIONES DEL PARTO ASOCIADAS CON LA MORTALIDAD PERINATAL

\begin{tabular}{|clcc|}
\hline No. & Complicación & Total & $* \%$ \\
\hline 1 & Parto pretérmino & 82 & 58.9 \\
2 & Parto por cesárea & 69 & 49.6 \\
3 & Parto de pelvis & 16 & 11.5 \\
4 & Accidentes del cordón & 5 & 3.6 \\
5 & Parto instrumentado & 4 & 2.9 \\
6 & Distocia de presentación & 3 & 2.1 \\
\hline
\end{tabular}

GRAFICA 19

AFECCIONES MATERNAS ASOCIADAS CON LA MORTALIDAD PERINATAL S/COMPONENTES

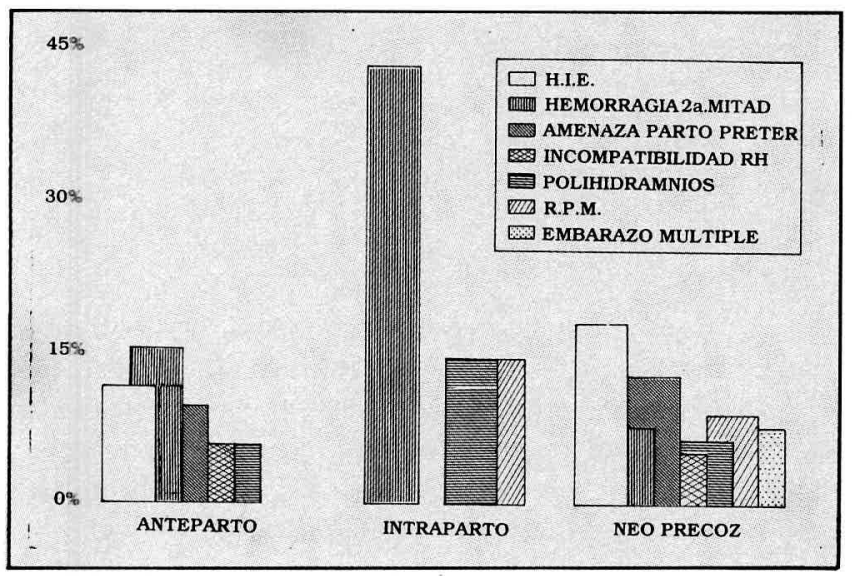

Alteraciones del parto asociadas con la mortalidad perinatal según sus componentes

El parto pretérmino en el componente anteparto e intraparto ocupó el primer lugar, y el segundo en el componente neonatal precoz en el que fue superado por el parto intervenido por operación cesárea, ésta ocupó un segundo lugar en los otros componentes. El tercer lugar en los tres componentes lo ocupó el parto de pelvis, 10 casos ocurrieron en la etapa neonatal precoz (Gráfica 20).

\section{Duración del trabajo de parto según compo- nentes}

La duración del trabajo de parto influye en la mortalidad intraparto y neonatal precoz; en el intraparto igual número de pacientes o no tuvieron o tuvieron trabajo de parto entre 2 y 6 horas, mientras en el componente neonatal el $43.04 \%$ no presentaron trabajo de parto y el $46.84 \%$ trabajaron entre 2 y 6 horas (Gráfica 21).
GRAFICA 20

\section{ALTERACIONES DEL PARTO ASOCIADAS CON LA MORTALIDAD PERINATAL S/COMPONENTES}

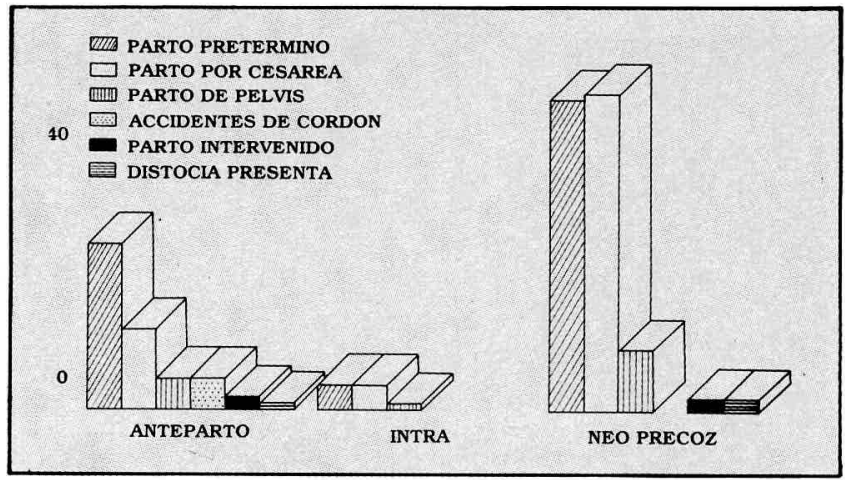

GRAFICA 21

DURACION DEL TRABAJO DE PARTO SEGUN COMPONENTES DE LA MORTALIDAD PERINATAL

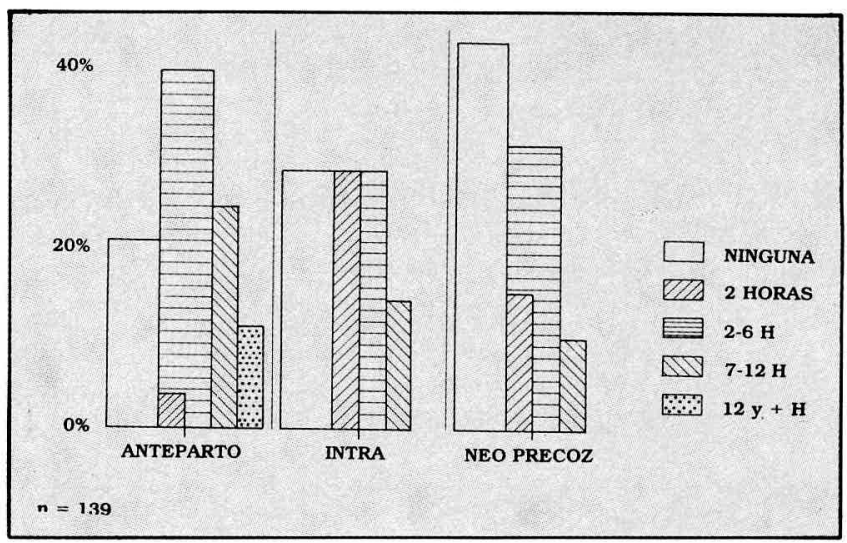

Tipo de presentación según componente de la mortalidad

La presentación cefálica es la mayoría en los tres componentes más del $80 \%$. La presentación podálica ocupa el lugar siguiente en los tres componentes. Otro tipo de presentación ocurrió en el $5.66 \%$ en la fase anteparto (Gráfica 22).

Tipo de parto según componentes de la mortalidad

Los casos de mortalidad antepartos fueron en un $71.7 \%$ atendidos por vía vaginal. El $24.53 \%$ de las cesáreas se realizaron en fetos muertos. En las muertes intraparto y neonatal temprana se intervinieron mediante cesárea el $57.14 \%$ y el $65.82 \%$ respectivamente. Partos instrumentados fueron muy escasos, en etapa neonatal el $2.53 \%$ (Gráfica 23). 
TIPO DE PRESENTACION DEL RN SEGUN COMPONENTES DE LA MORTALIDAD PERINATAL

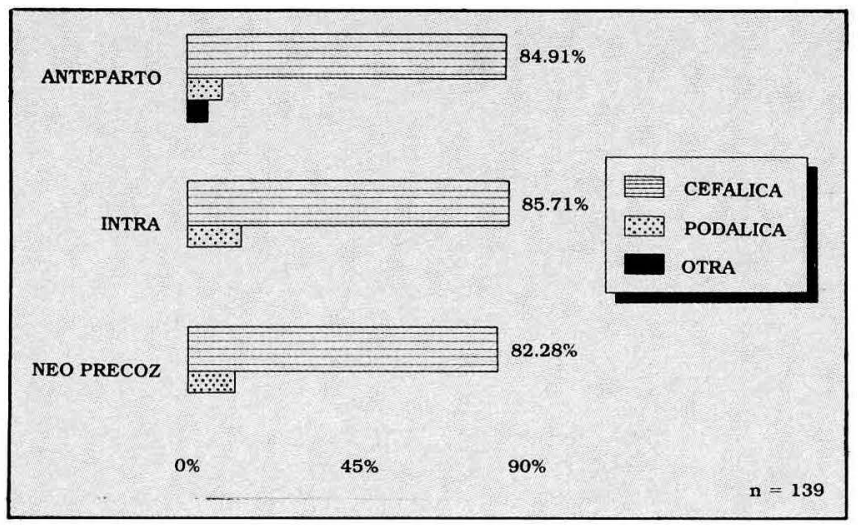

GRAFICA 23

TIPO DE PARTO SEGUN

COMPONENTES DE LA MORTALIDAD PERINATAL

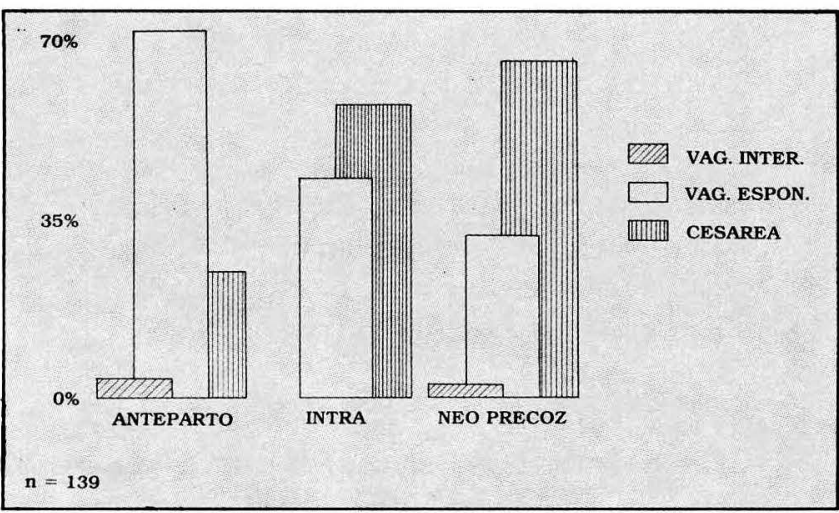

\section{Complicaciones del recién nacido asociadas} con la mortalidad perinatal

El predominio en los últimos cinco años ha sido del recién nacido pretérmino, dos de cada tres niños presentaron esta situación, el 58.9\% malformaciones congénitas en 34 de los 139 bebés muertos para un $24.5 \%$, síndrome de dificultad respiratoria en 28 , para un $20.1 \%$. Otras complicaciones, en número menor, se aprecian en la Tabla 3.

\section{Afecciones del neonato asociadas con la mor- talidad perinatal según componentes}

En cada componente se aprecia como predomina el recién nacido pretérmino; en el neonatal precoz el segundo lugar lo ocupa las malformaciones congénitas, y muy cerca, en el mismo componente, se destaca el síndrome de dificultad respiratoria (Gráfica 24).
COMPLICACIONES DEL RECIEN NACIDO ASOCIADAS CON LA MORTALIDAD PERINATAL

\begin{tabular}{|rlcr|}
\hline No. & \multicolumn{1}{c}{ Complicación } & Total & $* \%$ \\
\hline 1 & Pretérmino & 82 & 58.9 \\
2 & Malformaciones congénitas & 34 & 24.5 \\
3 & Sind. dificultad respiratoria & 28 & 20.1 \\
4 & Neumotórax-Neumomediastino & 12 & 8.6 \\
5 & Hemorragia cerebral & 9 & 6.5 \\
6 & Sufrimiento fetal intraparto & 8 & 5.8 \\
7 & Enf. Hemolítica por RH & 5 & 3.6 \\
8 & Sufrimiento fetal anteparto & 4 & 2.9 \\
9 & Hidrops fetalis & 3 & 2.2 \\
10 & Sepsis in útero o neonatal & 3 & 2.2 \\
$* \mathrm{n}=139$ & & \\
\hline
\end{tabular}

GRAFICA 24

AFECCIONES DEL NEONATO ASOCLADAS CON LA MORTALIDAD PERINATAL S/COMPONENTES

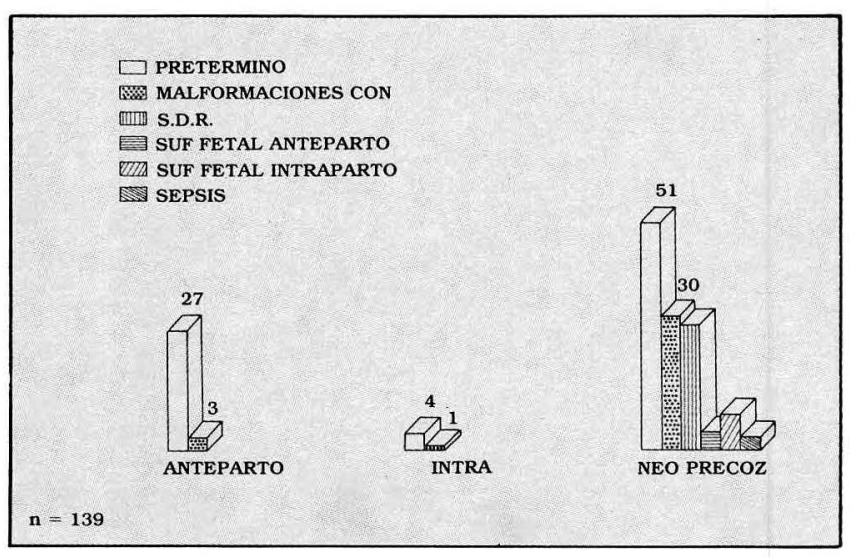

Primeras causas de mortalidad perinatal (según clasificación internacional de 999 causas)

La primera causa de muerte perinatal con el $24.5 \%$, una cuarta parte, fueron las malformaciones congénitas, el segundo lugar con el $20.1 \%$ lo ocupó el síndrome de dificultad respiratoria y, en tercer lugar el abruptio de placenta con un $10 \%$, estas tres causas representan el $54.6 \%$ del origen de las muertes (Tabla 4 ).

\section{Primeras causas de muerte perinatal según} edad de la madre

Al dividir en tres grupos de edad, menores de 18 años, de 19 a 34 y mayores de 35 años, se advierte en el segundo de ellos, un predominio del síndrome de dificultad respiratoria seguido del abruptio y el neumotórax. En las pacientes mayores de 35 años se aprecia al abruptio como causa dominante (Gráfica 25). 
TABLA 4

PRIMERAS CAUSAS DE MORTALIDAD PERINATAL SEGUN 999 CAUSAS

\begin{tabular}{|clcr|}
\hline No. & \multicolumn{1}{c}{ Causas } & Total & $* \%$ \\
\hline 1 & Malformaciones congénitas & 34 & 24.5 \\
2 & Sind. dificultad respiratoria & 28 & 20.1 \\
3 & Abruptio de placenta & 14 & 10.0 \\
4 & Neumotórax-Neumomediastino & 12 & 8.6 \\
5 & Hemorragia cerebral & 9 & 6.5 \\
6 & Sufrimiento fetal intraparto & 8 & 5.8 \\
7 & Enf. Hemolítica por RH & 5 & 3.6 \\
8 & Accidentes de cordón & 5 & 3.6 \\
9 & Sufrimiento fetal anteparto & 4 & 2.9 \\
10 & Hidrops fetalis & 3 & 2.2 \\
11 & Sepsis in úteroo neonatal & 3 & 2.2 \\
\hline
\end{tabular}

GRAFICA 25

\section{CAUSAS DE MORTALIDAD PERINATAL} SEGUN EDAD DE LA MADRE

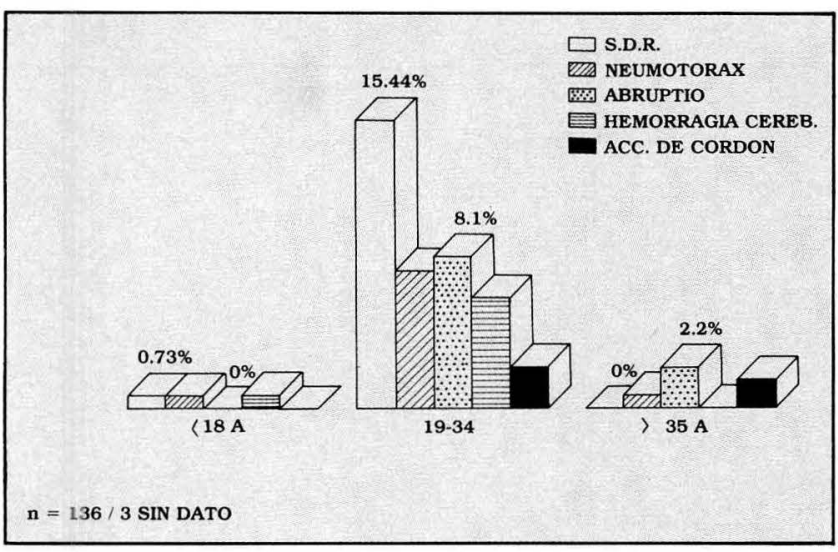

Primeras causas de mortalidad perinatal según atención prenatal

De 25 pacientes que no recibieron atención prenatal, a 5 no se les determinó causa; el parto pretérmino fue la primera causa en el $40 \%$ de los casos síndrome de dificultad respiratoria en el $20 \%$ de ellos y con el $12 \%$ se encontraron la hipertensión inducida por la gestación, embarazo múltiple, abruptio y malformaciones congénitas (Tabla 5).

\section{Primeras causas de mortalidad perinatal se- gún edad gestacional}

En el período pretérmino el síndrome de dificultad respiratoria ocupa el primer lugar con un $29.3 \%$ y el neumotórax un segundo lugar con $8.6 \%$. En la etapa a término el abruptio con el $20.4 \%$ está a la cabeza como causa de muerte perinatal, segundo y tercer lugar para el neumotórax y el síndrome de dificultad respiratoria con el $9.2 \%$ y el $7.4 \%$ respectivamente (Tabla 6 ).
TABLA 5

MORTALIDAD PERINATAL SEGUN ATENCION PRENATAL

\begin{tabular}{|lcc|}
\hline \multicolumn{1}{|c|}{ Causas } & No. & $\%$ \\
\hline Pretérmino & 10 & 40 \\
Sind. dificultad respiratoria & 5 & 20 \\
Hipertensión Ind. Emb. & 3 & 12 \\
Emb. Múltiple & 3 & 12 \\
Abruptio & 3 & 12 \\
Malformaciones cong. & 3 & 12 \\
Enf. Hemolítica del RN & 2 & 8 \\
Sin Causa & 5 & 20 \\
\hline
\end{tabular}

TABLA 6

\section{CAUSAS DE MORTALIDAD PERINATAL} SEGUN EDAD GESTACIONAL

\begin{tabular}{|c|c|c|c|c|c|}
\hline No. & $\begin{array}{l}\text { Pretérmino } \\
(28-32 \mathrm{~S})\end{array}$ & $\%$ & $\begin{array}{l}\text { Término } \\
(37-42 \mathrm{~S})\end{array}$ & $\%$ & $\begin{array}{c}\text { Post-term } \\
(>42 S)\end{array}$ \\
\hline 1 & S.D.R. & 29.3 & Abruptio & 20.4 & \\
\hline 2 & Neumotórax & 8.6 & Neumotórax & 9.2 & \\
\hline 3 & Hemor Cereb. & 7.3 & S.D.R. & 7.4 & \\
\hline 4 & S.F. Intra-P & 4.9 & Acc. cordón & 7.4 & \\
\hline 5 & Enf. Hemolítica & 4.9 & S.F. Intra-P & 7.4 & \\
\hline & OTROS & 45.2 & & 48.2 & \\
\hline
\end{tabular}

Primeras causas de mortalidad perinatal según tipo de parto

Sea el parto vaginal espontáneo o por operación cesárea el síndrome de dificultad respiratoria y el abruptio ocuparon los dos primeros lugares, si el parto fue abdominal la hemorragia cerebral acaeció en el $10.1 \%$ y si fue vaginal espontáneo ésta ocurrió en el 3\% (Tabla 7).

TABLA 7

\section{CAUSAS DE MORTALIDAD PERINATAL SEGUN TIPO DE PARTO}

\begin{tabular}{|clclccc|}
\hline No. Cesárea & & $\%$ & Espontáneo & $\%$ & Inter. & $\%$ \\
\hline I & S.D.R. & 26.1 & S.D.R. & 15.1 & Neumo & 25 \\
2 & Abruptio & 11.6 & Abruptio & 12.1 & & \\
3 & Hemor. Cereb. & 10.1 & Acc. cordón & 7.6 & & \\
4 & S.F. Intra-P & 10.1 & Neumotórax & 6.1 & & \\
5 & Neumotórax & 10.1 & Hemor. Cereb. & 3.0 & & 75 \\
\hline
\end{tabular}

Primeras causas de mortalidad perinatal según peso al nacer

Los niños de menos de 1.500 gramos presentan en el $47.5 \%$ las consecuencias de su inmadurez pulmonar y enseguida otra complicación de su prematurez, la hemorragia cerebral. En el rango de 1.500 a 2.499 gramos sigue ocupando la vanguardia las complicaciones de inmadurez pulmonar (Tabla 8). En los siguientes rangos, con peso mayor de 2.500 gramos, el abruptio ocupa el primer puesto, y las complicaciones pulmonares un lugar secundario (Tabla 9). 
TABLA 8

CAUSAS DE MORTALIDAD PERINATAL SEGUN PESO AL NACER

\begin{tabular}{|lllll|}
\hline & $<$ de 1.500 gr. & & $1.500 \mathrm{a}$ & \\
No. & & $\%$ & $2.499 \mathrm{gr}$ & $\%$ \\
\hline I & S.D.R. & 37.5 & S.D.R. & 21.6 \\
2 & Neumotórax & 10.0 & Abruptio & 7.9 \\
3 & Hemor. Cereb. & 7.5 & S.F. Intra-P & 7.9 \\
4 & Abruptio & 5.0 & Neumotórax & 7.9 \\
5 & Enf. Hemolítica & 2.5 & Acc. cordón & 5.9 \\
\hline
\end{tabular}

TABLA 9

CAUSAS DE MORTALIDAD PERINATAL SEGUN PESO AL NACER

\begin{tabular}{|cllll|}
\hline No. & $\begin{array}{l}2.500 \mathrm{a} \\
3.499 \mathrm{gr}\end{array}$ & $\%$ & $\begin{array}{l}3.500 \mathrm{y} \\
\text { más gr }\end{array}$ & $\%$ \\
\hline 1 & Abruptio & 17.9 & Abruptio & 14.3 \\
2 & Neumotórax & 10.2 & & \\
3 & S.F. Intra-P & 10.2 & & \\
4 & S.D.R. & 5.1 & & \\
5 & Acc.cordón & 5.1 & & 85.7 \\
\hline
\end{tabular}

$\mathrm{n}=137 / 2$ SIN DATOS

\section{Anomalías congénitas asociadas con la mor- talidad perinatal}

Treinta y cuatro de los casos de mortalidad perinatal correspondieron a anomalías congénitas incompatibles con la vida, seis de ellos presentaron malformaciones múltiples. Representan el $24.5 \%$ del grupo total, se aprecia su constante prevalencia a través de los cinco años del estudio. Las cardiopatías, las alteraciones del sistema nervioso central y la anencefalia ocupan los primeros sitios (Gráfica 26).

La corrección de la tasa de mortalidad perinatal, descontando estas malformaciones, nos permite obtener un valor de 14.86 por mil en el quinquenio 85 a 89 .

\section{COMENTARIOS}

De una tasa de mortalidad perinatal de 22.4 por mil en el decenio 63-73 se ha disminuido a un 19.69 en el quinquenio $85-89$.

El embarazo pretérmino continúa como causa de primer orden en los fallecimientos perinatales, aunque dos de cada tres recién nacidos tuvieron un peso superior a los 1.500 gramos, y peso promedio cerca de los 2.000 gramos.

La mortalidad neonatal precoz estuvo por encima de la fetal tardía, y en ésta, la intraparto contribuyó en un mínimo porcentaje. En la mitad de los casos la defunción se presentó después del quinto minuto de vida.

Embarazo complicado con trastorno hipertensivo, amenaza de parto pretérmino o abruptio de placenta, generalmente se acompaña de muerte perinatal. El parto prematuro e intervenido por cesárea se asociaron en
GRAFICA 26

ANOMALIAS CONGENITAS ASOCIADAS A LA MORTALIDAD PERINATAL

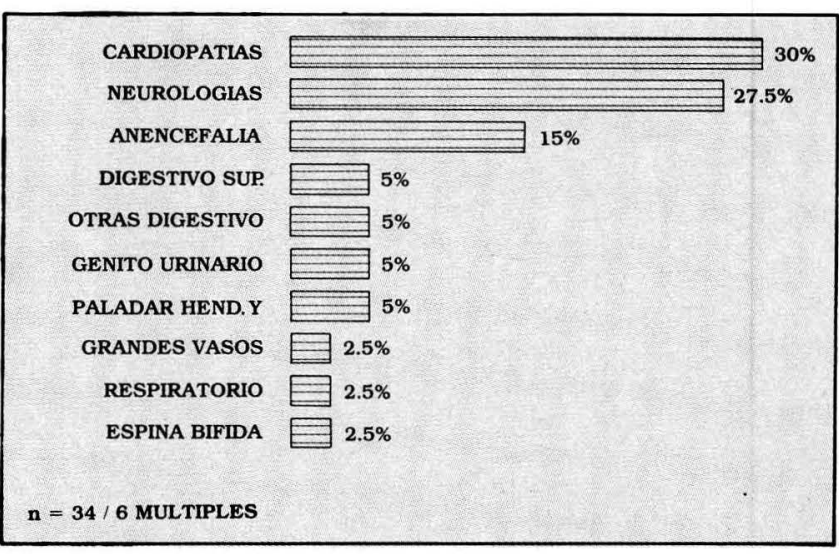

mayor número a estas muertes. Los niños pretérmino, con malformaciones y con alteraciones del aparato respiratorio participaron en mayor porcentaje en la muerte neonatal precoz. El diagnóstico respaldado por estudio patológico se obtuvo en el $37.41 \%$.

No por haber recurrido a la operación cesárea en la etapa neonatal precoz se mejoró la oportunidad de sobrevida al recién nacido, ya que cuando falleció por síndrome de dificultad respiratoria, en la mayoría, el parto se atendió por cesárea.

Un número inusualmente elevado de malformaciones congénitas ocupa la vanguardia en la lista de causas de mortalidad.

\section{CONCLUSIONES Y RECOMENDACIONES}

El índice de mortalidad perinatal encontrado continúa en nivel superior, comparativamente, a otras instituciones, en relación al número de partos atendidos.

Si en el control prenatal logramos detectar futuras madres con riesgo de trastorno hipertensivo, de amenaza y trabajo de parto pretérmino y brindamos un manejo apropiado a estas patologías, podremos contribuir a disminuir el número de muertes perinatales.

Al lograr el objetivo del párrafo anterior habremos disminuido notoriamente las pérdidas por prematurez y síndrome de dificultad respiratoria.

Mención aparte merece el índice elevado de malformaciones congénitas, situación que debe permitir la implementación de acciones para detección temprana y consejería genética.

Las causas más frecuentes de mortalidad perinatal halladas en este estudio son susceptibles de disminuirse con la adecuación de medidas preventivas y terapéuticas disponibles en nuestro medio. 
Los hallazgos aquí enumerados deben acceder al diseño e implementación de normas básicas para la atención prenatal y del parto, de acuerdo con las causas más frecuentes de mortalidad perinatal.
Debe estimularse la recolección permanente de la información, mediante el instrumento ya adecuado, con el fin de permitir evaluación periódica a las normas implementadas y correctivos a ellas si fuere el caso.

\section{BIBLIOGRAFIA}

1. ABUDU, O.; AKINKUBE, A. Clinical causes and clasification of perinatal mortality in lagos. Int $\mathrm{J}$ Gynecol Obstet 20(6): 443-7, 1982.

2. BALLAS, S.; HORNSTEIN, E. et al. Selective verus outine intrapartum monitoring: comparision of effects on perinatal outcome. Acta Obstet Gynecol Scand 59(4): 301-4, 1980.

3. BARNS, T. Obstetrics in the third world with particular reference to field research into delivery of maternal care to the comunity. En: Bourne C Recent advances in Obstetrics and Gynecology. Edimburgh; London, 1979; Churchill. Livingstone.

4. BOWES, W.A. Jr. A review of perinatal mortality in Colorado, 1971 to 1978 , and, its relationship to the regionalization of perinatal services. Am $\mathbf{J}$ Obstet Gynecol 141: 1045-52, 1981.

5. BYOLAN, P.O.; DRISCOLL, K. Improvement in perinatal mortality rate attributed to spontaneous preterm labor without use tocolytic agents. Am J Obstet Gynecol 145: 781-3, 1983.

6. DAIKOKU, N.H.; KALTREIDER, D.F.; JOHNSON, J.W.; SIMNONS, M.A. Premature rupture on membranes and preterm labor: Infection an perinatal mortality risks. Am J Public Health 71(1): 38-46, 1981.

7. DIAZ, R. Mortalidad perinatal en Bogotá, 1983-1984 Secretaría de Salud del Distrito Especial de Bogotá, Colombia, Cifras oficiales de certificados de defunción y de Natalidad. En proceso de publicación.

8. ELIZAGA, J.C. Métodos Demográficos para el estudio de la mortalidad. Centro Latino Americano de Demografia, serie E, No. 4, 1969.

9. ERKOLA, R.; KERO, P.; SEPP, A.L. et al. Monitoring perinatal Mortality by birth weight specific mortality rates. Int J Gynecol Obstet 20(3): 231-5, 1982.

10. FAUNDES, A.; HARDY, E.; DIAZ, J.; PINOTTI, J. Association of marital status and year of schooling with perinatal outcome; the influence of prenatal care as an intermediate variable. J Perinat Med 10(2): 105-13, 1982.

11. HOSKINS, E.M.; ELLIOT, E.; SHENNAN, A.T.; SKINDMORE, M.B.; KEITH, E. Out come of very lowbirth weight infants born at Perinatal Center. Am J Obstet Gynecol 145: 135-40, 1983.

12. INSULL, B.J.; MacLEAN, N.E.; BLANCHETTE, G. Perinatal Mortality in Southland. N $Z$ Med J 91(652): 40-2, 1980.

13. JUBIZ, A.; LONDOÑO, J. Morbilidad Perinatal. Rev colombiana de Obstet y Ginecol 35(6): 440-64, 1984.

14. KASS, E.H. Infectious disease and perinatal morbidity. Yale J Biol Med 55(3-4): 231-7, 1982.

15. KNUTZEN, V.K.; SHER, G. The mayor determinants of perinatal mortality in a large metropolitan Hospital. Results of a retrospective study. J Reprod Med 27(7): 395-400, 1982.

16. LANGER, A.; ARROYO P. La mortalidad perinatal en el Instituto Nacional de Perinatología, México. Bol Med Hosp Infant Mex 40(11), 1983.

17. LEE, K.; TSENG, P.; EIDELMAN, A.; KANDALL, S.;
GARTNER, L. Determinants of the neonatal mortality. Am J Dis Child 130: 842, 1976.

18. LINDGREN, L. Perinatal mortality and different socieconomic status. Acta Obstet Gynecol Scand 90(1): 21-6, 1981.

19. LOPEZ, G.; RIAÑO, G. Atención del parto Hospitalario en Colombia primeros hallazgos. Monografías de la CCRP; Vol 11; Junio 1979.

20. NAEYE, R.L. Abruptio Placentae and Placenta previa; Frecuency, perinatal mortality, and cigarette smoking. Obstet Gynecol 55(6): 701-4, 1980.

21. OCHOA, G. Mortalidad perinatal. Antioquia Médica 8(1):2-53, 1958.

22. OCHOA, G. et al. Resultados perinatal. Escuela Nacional de salud pública y facultad de medicina, Universidad de Antioquia. OMS/OPS. Informe preliminar.

23. O'DRISCOLL, K.; FOLEY, M. Correlation of decrease in perinatal mortality and increase in cesarean section rates. Obstet Gynecol 6(1): 1-5, 1983.

24. OMS. La prevención de la mortalidad perinatal. (Informe de un seminario). Cuadernos de salud pública No. 42 . Ginebra 1972.

25. OPS-OMS. Clasificación internacional de enfermedades. Vol 1-1975.

26. OPS. Manual sobre enfoque de riesgo en la atención materno infantil. No. 7, 1986.

27. PAEZ, R.; ACERO, U.; URIBE, H. Mortalidad Perinatal. Rev Colombiana de Obst y Ginecol 25(5): 249-58, 1974.

28. PERASON, J.W. Cesarean section and perinatal mortality. A nine year experience in a city country Hospital. Am J Obstet Gynecol 148(2): 155-9, 1984.

29. PERKINS, R.P. The neonatal significance of selected perinatal events. Among infants of low birth weight II. The influence of ruptured membranes. Am $\mathrm{J}$ Obstet Gynecol 142(1):7-16, 1982.

30. RODRIGUEZ, A.; MUNOZZ, L.A.; TAMARA, A.; LAGOS, M. Mortalidad Perinatal estudio de muestreo Instituto Materno Infantil de Bogotá.

31. Salud Perinatal, Boletín del Centro Latinoamericano de Perinatología y Desarrollo Humano. Vol 2 No. 4 de 1985.

32. Salud Perinatal, Boletín del Centro Latinoamericano de Perinatología y Desarrollo Humano. Vol 1 No. 3 de 1984.

33. STUBLEFIELD, P.G.; BEREK, J.S. Perinatal mortality in term and post-term births. Obstet Gynecol 56(6): 67682, 1980.

34. TERRIN, M.; MEYER, M.B. Birth weight especific rates as a bias in the effects of smoking and other perinatal hazzards. Obstet Gynecol 58(5): 636-8, 1981.

35. URIZA, G.; BARRAGAN, J. et al. Mortalidad perinatal. Estudio cooperativo institucional. Rev Colombiana Obst y Ginecol 37(2): 91-107, 1986.

36. URIZA, G.; BARRAGAN, J. et al. Estudio de la mortalidad perinatal en 21 instituciones de Colombia. Rev Colombiana Obst y Ginecol 39(2): 74-104, 1988.

37. WIGGLESWORTH, J. Perinatal Pathology Vol. 15 the series 1984 by W.B. Sannders Company. MPPLS. 